\title{
Bounds on the shear load of cohesionless granular matter
}

\author{
Wouter G. Ellenbroek ${ }^{1}$ and Jacco H. Snoeijer ${ }^{2}$ \\ ${ }^{1}$ Instituut-Lorentz, Universiteit Leiden, Postbus 9506, 2300 RA Leiden, The \\ Netherlands \\ ${ }^{2}$ School of Mathematics, University of Bristol, University Walk, Bristol BS8 \\ 1TW, United Kingdom
}

\begin{abstract}
We characterize the force state of shear-loaded granular matter by relating the macroscopic stress to statistical properties of the force network. The purely repulsive nature of the interaction between grains naturally provides an upper bound for the sustainable shear stress, which we analyze using an optimization procedure inspired by the so-called force network ensemble. We establish a relation between the maximum possible shear resistance and the friction coefficient between individual grains, and find that anisotropies of the contact network (or the fabric tensor) only have a subdominant effect. These results can be considered the hyperstatic limit of the force network ensemble and we discuss possible implications for real systems. Finally, we argue how force anisotropies can be related quantitatively to experimental measurements of the effective elastic constants.

PACS numbers: 45.70.Cc, 05.40.-a, 46.65.+g
\end{abstract}

\section{Introduction}

An assembly of cohesionless granular matter, in which there is no attraction between grains, can only exist when held together by an external pressure [1]. The distribution of these confining forces throughout the material is a complex process that involves a highly inhomogeneous network of contact forces [2, 3, 4, 5]. Force networks as shown in figure 1h are typical for a broad variety of amorphous systems like foams, colloids and emulsions, and play a crucial role for understanding the macroscopic mechanical properties [6, 7, 8].

A robust feature of these "jammed" materials is that they can sustain a certain amount of shear stress before failure [9, 10, 11, 12. There are many aspects that influence this shear resistance or internal friction of a granular material. A well known example is that wet sand can sustain much larger shear stresses than dry sand, due to the presence of attractive liquid bridges 13, 14. The strength of the assembly is also enhanced by increasing intergrain friction or roughness of the grains. However, if one slowly increases the applied shear stress and follows the evolution of contact forces and grain locations, one encounters very complex collective phenomena. Before the system yields as a whole, there are non-adiabatic precursor events such as local rearrangements due to instability of subsets of grains [15, 16, 17. This will induce changes in fabric and coordination number, and it has remained a great challenge to understand how this couples back to the stress state [17, 18, 19, 20]. The conventional 

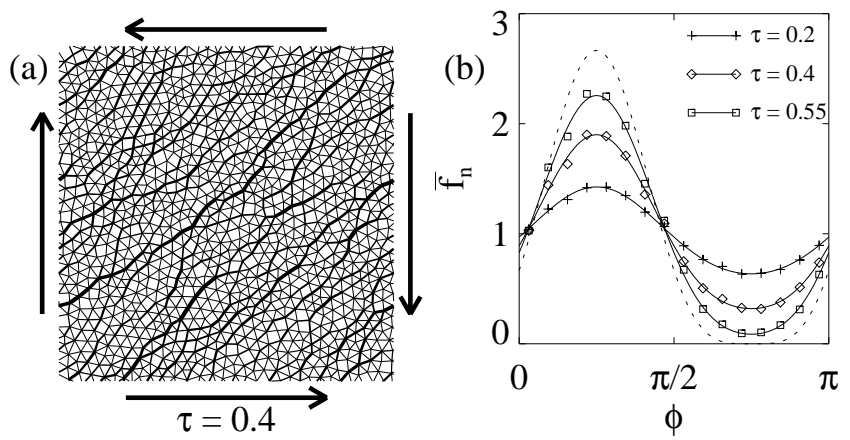

Figure 1. (a) Force network obtained from a numerical simulation of a strongly hyperstatic frictionless granular material (coordination number $z=$ 5.75) subjected to a shear stress $\tau=\sigma_{x y} / \sigma_{x x}$, using the "force network ensemble" 5] 21. The thicknesses of lines represent the strength of the forces. (b) Corresponding average contact force as a function of contact angle $\phi$ for this ensemble of force networks. Increasing the shear stress yields a modulation of $\bar{f}(\phi)$ that is accurately described by the form $1+2 \tau \sin 2 \phi+b_{2} \cos 4 \phi$. For large stress, $\bar{f}(\phi)$ approaches the limiting curve predicted by [19].

tool to study this problem is direct numerical simulation of the particle dynamics. While this provides valuable information on the micromechanics of sheared granular materials, it remains very difficult to distinguish the relative importance of contact and force anisotropies: both are evolving simultaneously.

Recently, a different strategy based on "ensembles of force networks" has been proposed to address this problem [21]. In this approach one investigates the statistics of all possible force network configurations that are mechanically stable, for a single fixed packing geometry of grains [1, 5, 22, 23. This allows explicit separation of the effects of forces and texture, e.g. by studying the force ensembles for packs of different fabric and coordination numbers. Consistent with direct simulations [16, the ensemble showed that packings close to the minimum isostatic coordination number can hardly support any stress whereas strongly hyperstatic packings (much higher coordination numbers) can sustain much more stress [21]. At the same time, the theory also provides a very realistic description of the force anisotropy, force fluctuations and response function [5, 21, 22, 23, 24, 25.

In this paper we derive upper bounds for the shear load of cohesionless granular media, for varying intergrain friction and fabric anisotropy. The analysis is based on an intriguing observation made in the force network ensemble for strongly hyperstatic packs. The most elementary manifestation of force anisotropy is the modulation of the average force $\overline{\mathbf{f}}(\phi)$, as a function of the contact orientation $\phi$. From figure $\mathbf{1}$, obtained from a two-dimensional frictionless system, it is clear that the force anisotropy is limited by the requirement that the normal component $\bar{f}_{n}(\phi) \geq 0$ for all $\phi$. This is due to the repulsive nature of the contact forces which require all $f_{i j} \geq 0$. Indeed, it was found that this simple criterion provides a very good approximation of the maximum shear stress achieved in the force network ensemble, in the limit of hyperstatic packs 21. It also gives a good prediction for the limiting form of $\overline{\mathbf{f}}(\phi)$ upon approaching the maximum load.

The quantity $\overline{\mathbf{f}}(\phi)$ thus provides crucial information on the force anisotropy and has recently been accessed experimentally for the first time [8]. To generalize the 


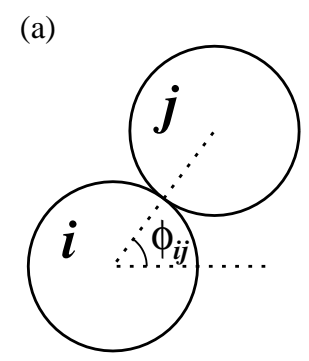

(b)

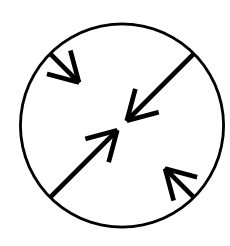

(c)

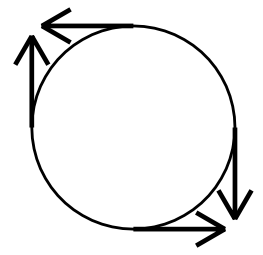

Figure 2. (a) Definition of contact orientation $\phi_{i j}$. (b,c) Illustration of the (average) bias of normal and tangential forces onto a particle due to the imposed shear stress.

arguments above to frictional systems, we have to translate the physical constraints for all individual contact forces,

- normal forces are purely repulsive, i.e. $f_{n} \geq 0$,

- tangential forces $f_{t}$ obey Coulomb's law of friction, $\left|f_{t}\right| \leq \mu f_{n}$,

to constraints for $\overline{\mathbf{f}}(\phi)$. Here, $\mu$ is the Coulomb friction coefficient of the individual contacts. We show how this yields nontrivial predictions for the maximum stress by finding the extreme forms of $\overline{\mathbf{f}}(\phi)$. While these maxima can probably not be reached in real systems, they describe the strongly hyperstatic limit of the force network ensemble, making it a well-defined analytical tool to investigate the influence of the micromechanical parameters on the effective macroscopic friction.

The optimization approach followed in the present paper is in the same spirit as the force network ensemble, in the sense that it deals with the question if a force network with given parameters can exist or not, rather than with the actual evolution of a sheared granular system. On the other hand, while force balance on each grain is explicitly taken into account in the force network ensemble, it plays no role in our analysis of $\overline{\mathbf{f}}(\phi)$. This is allowed in the strongly hyperstatic limit, but not for nearly isostatic packs. In the latter case the force networks are to a large extent determined by the conditions of force balance [5, 26, 27.

The relevant macroscopic quantity is the deviatoric stress, defined as $\tau=$ $\left(\sigma_{1}-\sigma_{2}\right) /\left(\sigma_{1}+\sigma_{2}\right)$, where the $\sigma_{i}$ denote the principal values of the stress tensor. We work in the coordinate frame where $\sigma_{x x}=\sigma_{y y}$, so we can express the deviatoric stress as $\tau=\sigma_{x y} / \sigma_{x x}$. For cohesionless systems one may naively expect that the ultimate shear stress is achieved when one principal direction becomes tensile, e.g. $\sigma_{2}=0$, which would lead to $\tau_{\max }=1$. By invoking realistic structures of $\overline{\mathbf{f}}(\phi)$ for granular packs, however, we show that the physics is in fact much more subtle, and that the real maximum is typically much lower than unity. Our main findings are that the effect of friction between grains is only mild: a typical Coulomb friction coefficient of $\mu=0.5$ increases $\tau_{\max }$ by only $16 \%$ as compared to the frictionless case. We also find that realistic anisotropies in the contact fabric hardly increase $\tau_{\max }$ and thus seem to play a subdominant role in real systems.

The paper is organized as follows. We first define the quantities that will be used to analyse the bounds, defined from the microscopic structure of grains and contacts, in relation to the macroscopic shear stress in section 2 The third section addresses the simplest case of isotropic, frictionless packings, and shows how the granularity of 
the material affects the maximally supportable shear stress. In sections 4 and 5 we explore the effects of intergrain friction and fabric anisotropies on the maximum shear stress. We conclude in section [6 where we argue how our approach can be applied to problems of anisotropic elasticity.

\section{From contact forces to macroscopic stress}

We consider two-dimensional packings of discs, so that the orientations of the contacts between particles $i$ and $j$ can be characterized by the angle (figure $2 a$ )

$$
\phi_{i j}=\arccos \left(\frac{x_{j}-x_{i}}{\left|\mathbf{r}_{j}-\mathbf{r}_{i}\right|}\right) .
$$

Here $\mathbf{r}_{i}$ denotes the position vector of particle $i$ and $x_{i}$ its $x$-coordinate. The key quantity that we will use to determine the maximum shear load is the average force $\overline{\mathbf{f}}(\phi)$ carried by all contacts of orientation $\phi$. Since bond directions have no polarity, the angle only assumes values $0 \leq \phi<\pi$, and the period of $\overline{\mathbf{f}}(\phi)$ is $\pi$. One can relate $\overline{\mathbf{f}}(\phi)$ to the stress tensor $\sigma$, using [28]

$$
\sigma_{\alpha \beta}=\frac{1}{V} \sum_{\{i j\}}\left(\mathbf{f}_{i j}\right)_{\alpha}\left(\mathbf{r}_{i j}\right)_{\beta} \equiv \frac{N_{c}}{V} \overline{\mathbf{f}_{\alpha} \mathbf{r}_{\beta}} .
$$

Here $\alpha, \beta$ label coordinate axes, $N_{c}$ is the number of contacts in the (two-dimensional) volume $V, \mathbf{r}_{i j}=\mathbf{r}_{j}-\mathbf{r}_{i}$, and $\mathbf{f}_{i j}$ is the force exerted on particle $j$ by particle $i$. The bar indicates average over all forces in $V$. We decompose the force vector in a normal component, $f_{\mathrm{n}, i j}$, and tangential components, $f_{\mathrm{t}, i j}$, as

$$
\begin{aligned}
& \left(\mathbf{f}_{i j}\right)_{x}=f_{\mathrm{n}, i j} \cos \phi_{i j}-f_{\mathrm{t}, i j} \sin \phi_{i j} \\
& \left(\mathbf{f}_{i j}\right)_{y}=f_{\mathrm{n}, i j} \sin \phi_{i j}+f_{\mathrm{t}, i j} \cos \phi_{i j} .
\end{aligned}
$$

The sign conventions are such that repulsive forces have positive $f_{\mathrm{n}, i j}$, while the tangential component is positive when pointing "counterclockwise" with respect to particle $i$ (see figure 2).

For large enough packings we can express the stress tensor in a statistical form, evaluating the average $\overline{\mathbf{f}_{\alpha} \mathbf{r}_{\beta}}$ from the probability to find a contact with force $\mathbf{f}_{i j}$ and centre-to-centre vector $\mathbf{r}_{i j}$. In terms of normal and tangential components, and with the observation that the forces are uncorrelated to the interparticle distance $\left|\mathbf{r}_{i j}\right|$ [21, 29], this involves the joint probability $P\left(f_{\mathrm{n}}, f_{\mathrm{t}}, \phi\right)$. We can explicitly factorize the contact angle probability $\Phi(\phi)$ to write

$$
P\left(f_{\mathrm{n}}, f_{\mathrm{t}}, \phi\right)=\Phi(\phi) P_{\phi}\left(f_{\mathrm{n}}, f_{\mathrm{t}}\right) .
$$

The distribution $P_{\phi}\left(f_{\mathrm{n}}, f_{\mathrm{t}}\right)$ has been introduced recently [21] and is properly normalized to unity. Hence, the probabilistic form of the stress tensor reads

$$
\begin{aligned}
\sigma_{\alpha \beta}= & \frac{\bar{r} N_{c}}{V} \int_{0}^{\pi} \mathrm{d} \phi \Phi(\phi) \int_{0}^{\infty} \mathrm{d} f_{\mathrm{n}} \int_{-\infty}^{\infty} \mathrm{d} f_{\mathrm{t}} \\
& \times P_{\phi}\left(f_{\mathrm{n}}, f_{\mathrm{t}}\right)\left[f_{\mathrm{n}} \mathcal{N}_{\alpha \beta}+f_{\mathrm{t}} \mathcal{T}_{\alpha \beta}\right] \\
= & \frac{\bar{r} N_{c}}{V} \int_{0}^{\pi} \mathrm{d} \phi \Phi(\phi)\left[\bar{f}_{\mathrm{n}}(\phi) \mathcal{N}_{\alpha \beta}+\bar{f}_{\mathrm{t}}(\phi) \mathcal{T}_{\alpha \beta}\right],
\end{aligned}
$$


where $\bar{r}$ denotes the average interparticle distance. The projection factors have been collected in tensors $\mathcal{N}_{\alpha \beta}$ and $\mathcal{T}_{\alpha \beta}$, written in matrix notation as

$$
\begin{aligned}
& \mathcal{N}_{\alpha \beta}=\left(\begin{array}{cc}
\cos ^{2} \phi & \cos \phi \sin \phi \\
\cos \phi \sin \phi & \sin ^{2} \phi
\end{array}\right) \\
& \mathcal{T}_{\alpha \beta}=\left(\begin{array}{cc}
-\cos \phi \sin \phi & -\sin ^{2} \phi \\
\cos ^{2} \phi & \cos \phi \sin \phi
\end{array}\right) .
\end{aligned}
$$

In the remainder, we will set the prefactor $\bar{r} N_{c} / V=1$ in (5).

The above analysis allows computing the stress from $\overline{\mathbf{f}}(\phi)$. To relate the force anisotropies to the shear stress, a common trick is to expand $\overline{\mathbf{f}}(\phi)$ in a Fourier series [19, 21, 29]

$$
\begin{aligned}
& \bar{f}_{\mathrm{n}}(\phi)=\sum_{k=1}^{N} a_{k} \sin 2 k \phi+\sum_{k=0}^{N} b_{k} \cos 2 k \phi \\
& \bar{f}_{\mathrm{t}}(\phi)=\sum_{k=1}^{N} c_{k} \sin 2 k \phi+\sum_{k=0}^{N} d_{k} \cos 2 k \phi .
\end{aligned}
$$

Because we are working in the frame where $\sigma_{x x}=\sigma_{y y}$, the principal axes of stress point in the directions $(1,1)$ and $(1,-1)$. These directions must then be lines of mirror symmetry, as is illustrated in figure [2] which makes that $a_{k}=d_{k}=0$ for even $k$ and $b_{k}=c_{k}=0$ for odd $k$.

For the moment, until the last section of the paper, we will consider the case where the fabric is isotropic so $\Phi(\phi)=1 / \pi$. In that case, inserting equations (89) in (5) and integrating yields

$$
\begin{aligned}
\sigma_{x x} & =b_{0} / 2 \\
\sigma_{y y} & =b_{0} / 2 \\
\sigma_{x y} & =\left(a_{1}+d_{1}\right) / 4 .
\end{aligned}
$$

All higher order terms in the expansion yield zero upon integration. Our main interest is the deviatoric stress, so we are free to choose the pressure scale as $\sigma_{x x}=\sigma_{y y}=1 / 2$, so that $\bar{f}=b_{0}=1$ and

$$
\tau=\frac{a_{1}+d_{1}}{2} .
$$

This relation reveals how an applied shear stress can be sustained through anisotropies in both the normal and frictional forces, via $a_{1}$ and $d_{1}$ respectively [29]. The strategy will be to explore the physical limitations of $a_{1}$ and $d_{1}$, which will provide a bound on $\tau$.

Note that due to the linearity of (2), the stress only couples to the first moment of the force distributions. This means that the stress does not depend on details of the probability $P(|\mathbf{f}|)$ 3, 4, 5, 7, 8. Also, the stress tensor contains no information on "force chains", i.e. the tendency of large forces the align in a correlated way. (2) does not invoke products of different $\mathbf{f}_{i j}$ so that spatial force-force correlations do not come into play.

\section{Frictionless packings}

We start out with packings of frictionless particles, for which the problem of the maximum possible deviatoric stress is relatively straightforward. In this case a bound 
on $\tau$ emerges from the purely repulsive nature of the forces: all contacts have $f_{\mathrm{n}, i j} \geq 0$, so certainly the averages should obey

$$
\bar{f}_{\mathrm{n}}(\phi) \geq 0
$$

for all values of $\phi$. This condition obviously forms a serious restriction on the amplitude of the force anisotropy, and we show how this yields an upper limit on $\tau$. We show that this maximum value $\tau_{\max }$ depends on the number $N$ of terms included in the Fourier series of (8): even though the higher order terms do not contribute to the stress, they enable $f_{\mathrm{n}, i j}$ to reach more extreme shapes. We first explore the case $N=2$, which is the relevant case for real systems (see also Appendix A). Then we discuss the problem for arbitraty $N$, which provides some additional insights.

\subsection{Realistic $\bar{f}_{\mathrm{n}}(\phi)$}

For the frictionless case we have from (13)

$$
a_{1}=2 \tau, \quad d_{1}=0,
$$

so that (8) becomes

$$
\bar{f}_{\mathrm{n}}(\phi)=1+2 \tau \sin 2 \phi+b_{2} \cos 4 \phi+\cdots
$$

Let us first consider the simplest case, in which we truncate after the lowest anistropic term, i.e. $\bar{f}_{\mathrm{n}}(\phi)=1+2 \tau \sin 2 \phi$, so that $\tau$ is the only free parameter. For positive $\tau$ this function has a minimum in $\phi=3 \pi / 4$, which touches $\bar{f}_{\mathrm{n}}(\phi)=0$ for $2 \tau=1$. Hence, $\tau_{\max }=1 / 2$. In the same way we could derive $\tau_{\min }=-1 / 2$. From now on we will only consider positive $\tau$, without loss of generality.

From the numerical result of figure 10 it is clear that the modulation does not stay symmetric around $\bar{f}_{\mathrm{n}}(\phi)=1$ for large values of $\tau$. This indicates a significant contribution of the type $\cos 4 \phi$. This is the highest order term that we can expect to be relevant in granular matter, because steric exclusion between the grains sets a lower limit to the width of peaks in $\bar{f}_{\mathrm{n}}(\phi)$. This is explained in more detail in Appendix A

We thus focus on the case $N=2$ in the expansion (8). The optimization problem involves two free parameters, $\tau$ and $b_{2}$. We are free to vary $b_{2}$ in such a way as to facilitate a maximum $\tau$, under the constraint that $\bar{f}_{\mathrm{n}}(\phi) \geq 0$ for all $\phi$. We furthermore demand that $\bar{f}_{\mathrm{n}}(\phi)$ evolves monotonically between the principal directions at $\phi=\pi / 4$ and $\phi=3 \pi / 4$. This implies that the minimum of $\bar{f}_{\mathrm{n}}(\phi)$ should stay at $\phi=3 \pi / 4$, as it is the case in the numerics of figure 1 b. Physically, this means that the first contacts to break are those oriented in the direction in which the material is stretched. While the expansion ensures that there is an extremum in $\phi=3 \pi / 4$, this extremum only remains a minimum if

$$
\left.\frac{\partial^{2}}{\partial \phi^{2}} \bar{f}_{\mathrm{n}}(\phi)\right|_{\phi=\frac{3 \pi}{4}}=8 \tau+16 b_{2} \geq 0 .
$$

The value of $\bar{f}_{\mathrm{n}}(\phi)$ in this minimum must satisfy

$$
\bar{f}_{\mathrm{n}}(3 \pi / 4)=1-2 \tau-b_{2} \geq 0 .
$$




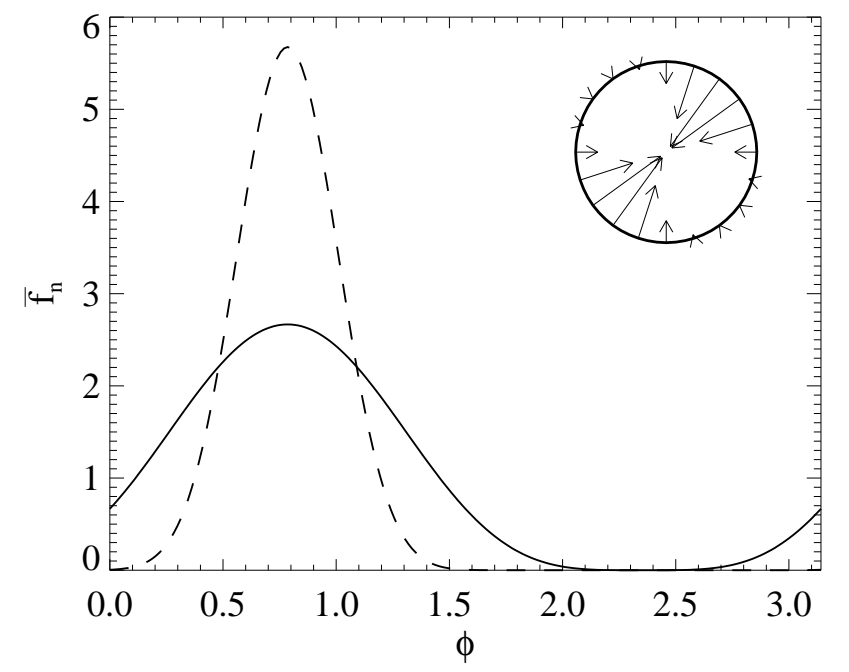

Figure 3. The optimized $\bar{f}_{\mathrm{n}}(\phi)$ for $N=2$ (solid), $N=10$ (dashed). The figure in the corner illustrates the corresponding average force exerted on a particle for various contact orientations $(N=2)$.

This defines a linear program with parameters $\tau$ and $b_{2}$, two inequalities, and the objective to maximize $\tau$. The solution is easily found to be $\ddagger$

$$
\begin{aligned}
& \tau_{\max }=\frac{2}{3} \\
& b_{2}=-\frac{1}{3} .
\end{aligned}
$$

figure 10 illustrates the relevance of this bound for strongly hyperstatic packings: the numerical $\bar{f}_{\mathrm{n}}(\phi)$ (taken from [21]) indeed approaches the limiting form (dashed curve)

$$
\bar{f}_{\mathrm{n}}(\phi)=1+\frac{2}{3} \sin 2 \phi-\frac{1}{3} \cos 4 \phi
$$

So indeed, the system is able to organize the forces in such a manner as to optimize the sustained shear stress.

\subsection{The limit $N \rightarrow \infty$ and tensile stresses}

Although Fourier terms with $k \geq 3$ do not play a role in granular systems, it is insightful to study the general case where we truncate the series at arbitrary order $N$. The expansion now contains $N$ free coefficients that we need to fix, in order to optimize $\tau$. In the case $N=2$, we invoked the condition $\partial^{2} \bar{f}_{\mathrm{n}} / \partial \phi^{2} \geq 0$ at $\phi=3 \pi / 4$ to ensure that the minor principal axis remains a minimum of $\bar{f}_{\mathrm{n}}(\phi)$, and taking $\partial^{2} \bar{f}_{\mathrm{n}} / \partial \phi^{2}=0$ turned out to maximize $\tau$. A nonzero $k=3$ term allows to also fix $\partial^{4} \bar{f}_{\mathrm{n}} / \partial \phi^{4}$ in such a way that $\tau$ becomes even larger. The upshot of adding more terms is that we can make $\bar{f}_{\mathrm{n}}(\phi)$ as flat (and as close to zero) as possible around $\phi=3 \pi / 4$, where the contribution to the overlap with $\sin 2 \phi$ is negative. Every time we have to verify that

$\ddagger$ Actually, because for these values of the parameters the second derivative vanishes in $\phi=3 \pi / 4$, we have to check that the fourth derivative is positive to be sure that the extremum is a minimum, which is indeed the case. 
the first nonzero Taylor coefficient when expanding around $\phi=3 \pi / 4$ is positive, so that we are indeed dealing with a minimum.

The general scheme is thus that adding the term of order $k$ generates an additional condition that $\partial^{(2 k-2)} \bar{f}_{\mathrm{n}} / \partial \phi^{(2 k-2)}=0$. For general $N$, this yields the following set of linear equations:

$$
\left.\frac{\partial^{2 l}}{\partial \phi^{2 l}} \bar{f}_{\mathrm{n}}(\phi)\right|_{\phi=\frac{3 \pi}{4}}=0 \quad \text { for all } \quad l=0,2, \cdots, N-1 .
$$

In Appendix B we show that this linear problem for the Fourier coefficients can be inverted analytically, yielding a remarkably simple result for the maximum $\tau$, namely

$$
\tau_{\max }(N)=\frac{N}{N+1}
$$

Interestingly, this reveals an ultimate (mathematical) maximum shear stress $\tau_{\max }=1$. We plotted the optimized $\bar{f}_{\mathrm{n}}(\phi)$ for various values of $N$ (figure 31): clearly, $\bar{f}_{\mathrm{n}}(\phi)$ evolves towards the extreme case of a Dirac $\delta$-peak in the limit $N \rightarrow \infty$. As already mentioned in the introduction, this condition of $\tau=1$ precisely corresponds to the point where the minor principal axis becomes tensile. We thus conclude that, due to the finite width of $\bar{f}_{\mathrm{n}}(\phi)$, the maximum stress for a frictionless packing lies well below the point where the global stress unavoidably develops a tensile direction. We have argued in Appendix A that this finite width is in fact due to steric exclusion between neighbouring grains. This illustrates how the discrete nature of the assembly has an important effect on global properties.

\section{Frictional packings: $\tau_{\max }(\mu)$}

The presence of frictional forces provides an additional degree of freedom to develop anisotropic stresses: Equation (13) shows that the total deviatoric is the sum of the (lowest order) anistropies of normal and tangential forces. It is clear that this will enhance the ability to sustain a large external load. However, there is again a bound on the force anisotropies, now due to Coulomb's law for individual contacts, i.e. $\left|f_{\mathrm{t}, i j}\right| \leq \mu f_{\mathrm{n}, i j}$, where $\mu$ is the microscopic Coulomb friction coefficient. Since this condition should hold for any pair of grains, it certainly holds for the averages:

$$
\left|\bar{f}_{\mathrm{t}}(\phi)\right| \leq \mu \bar{f}_{\mathrm{n}}(\phi) .
$$

This condition is illustrated in figure 4 In this section we derive how $\tau_{\max }$ depends on the value of $\mu$, again using the Fourier expansions of $\bar{f}_{\mathrm{n}}(\phi)$ and $\bar{f}_{\mathrm{t}}(\phi)$ up to $N=2$.

\subsection{The optimization problem}

From (13) we can express

$$
a_{1}=2 \tau-d_{1}
$$

so that

$$
\begin{aligned}
& \bar{f}_{\mathrm{n}}(\phi)=1+\left(2 \tau-d_{1}\right) \sin 2 \phi+b_{2} \cos 4 \phi, \\
& \bar{f}_{\mathrm{t}}(\phi)=d_{1} \cos 2 \phi+c_{2} \sin 4 \phi .
\end{aligned}
$$

Let us now take a value of $\tau$ slightly above the frictionless limit $2 / 3$. The prefactor in front of the $\sin 2 \phi$ term can now be lowered due to $d_{1}$, i.e. due to the presence of 


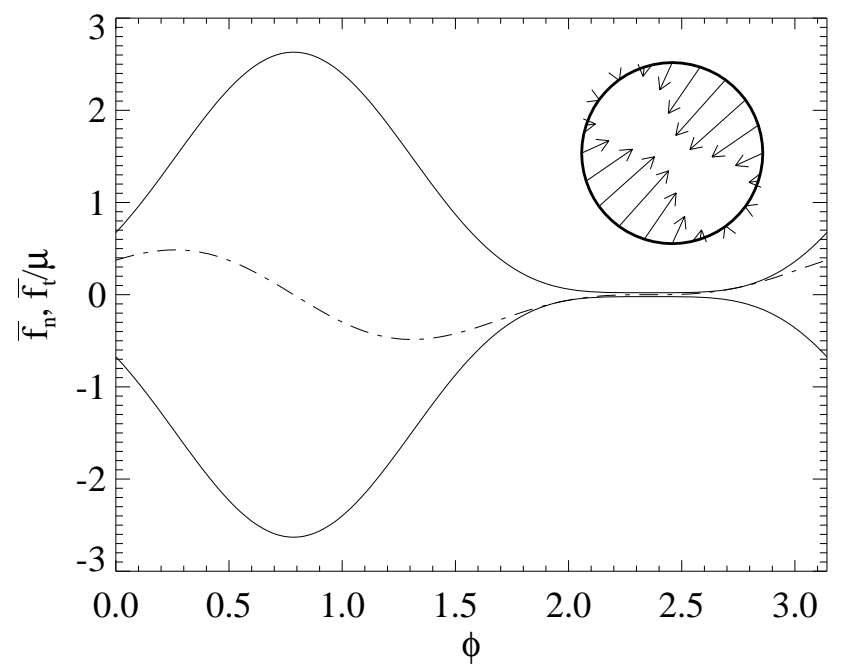

Figure 4. The frictional optimization problem for $\mu=0.28$. The solid lines represent $\pm \bar{f}_{\mathrm{n}}(\phi)$, the dash-dotted line $\bar{f}_{\mathrm{t}}(\phi) / \mu$, which has to satisfy (22). The figure in the corner illustrates the average force exerted on a frictional particle for various contact orientations. Note that these forces now have tangential components.

friction. If we put $\tau$ far above $2 / 3$, however, one requires a relatively large $d_{1}$. The value of $d_{1}$ is bounded by the condition of (22), so that not all $\tau$ can be reached.

To determine the maximum value of $\tau$ as a function of $\mu$, we have to specify acceptable values of the higher order coefficients $b_{2}$ and $c_{2}$, which do not contribute to the stress tensor. As we did in the frictionless case, we demand that the function $\bar{f}_{\mathrm{n}}(\phi)$ evolves monotonically between major and minor directions, so that it has only one maximum (in the major direction) and only one minimum (in the minor direction). A similar requirement is imposed on $\bar{f}_{\mathrm{t}}(\phi)$ : the average tangential force only changes sign

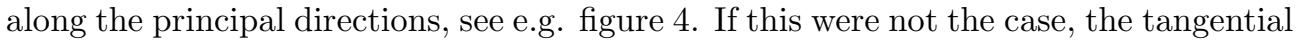
forces would swap from clockwise to counterclockwise and back in between the major and minor directions. Such a spontaneous symmetry breaking would introduce a very nongeneric organization of forces within the packing. These conditions put bounds on the second order coefficients,

$$
\begin{aligned}
& -\frac{2 \tau-d_{1}}{4} \leq b_{2} \leq \frac{2 \tau-d_{1}}{4}, \\
& -\frac{d_{1}}{2} \leq \quad c_{2} \leq \frac{d_{1}}{2} .
\end{aligned}
$$

We have numerically solved this optimization problem by varying all possible values of the parameters $\tau, d_{1}, b_{2}, c_{2}$, within the ranges imposed by equations (22)26127). The results are shown in figure 5 Surprisingly, the dependence on $\mu$ is relatively weak. In the paragraph below we obtain the analytical result,

$$
\tau_{\max }=\frac{1+\sqrt{1+3 \mu^{2}}}{3},
$$

which is derived for $1-\frac{1}{2} \sqrt{2} \leq \mu \leq 1$. However, figure 5 shows that this is a very good approximation outside this range as well. 


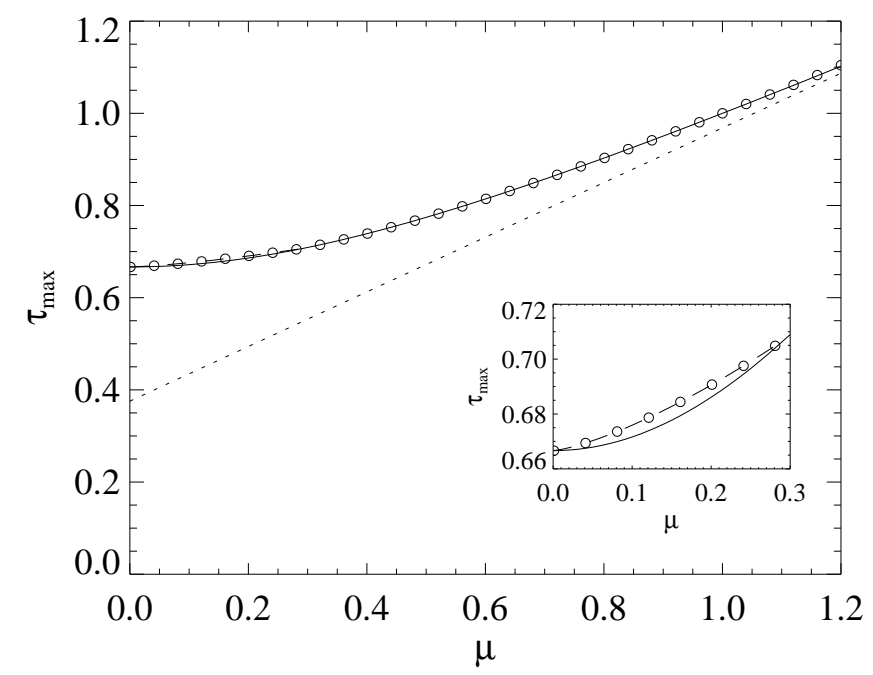

Figure 5. The optimized $\tau(\mu)$ for frictional packings with isotropic fabric. The circles are numerical data. The solid line is equation [28). The dashed line is the analytic result for $\mu \leq 1-\frac{1}{2} \sqrt{2} \approx 0.29$. The dotted line is the asymptote $\tau \propto 0.593 \mu$ as $\mu \rightarrow \infty$. The inset shows an enlargement of the small $\mu$ region.

\subsection{Analytical solution of $\tau(\mu)$}

The set of parameters $\tau, d_{1}, b_{2}, c_{2}$ that corresponds to the maximum value of $\tau$ obviously corresponds to functions $\bar{f}_{\mathrm{t}}(\phi)$ and $\mu \bar{f}_{\mathrm{n}}(\phi)$ that are tangent in at least one point. We will now derive the optimal set of parameters for the case there is a tangent point in the interval $\frac{7 \pi}{8} \leq \phi \leq \pi$, and then determine for what range of $\mu$ this is the case.

In the interval $\left[\frac{7 \pi}{8}, \pi\right]$, the $\cos 2 \phi$ in $\bar{f}_{\mathrm{t}}(\phi)$ is positive and the $\sin 4 \phi$ is negative. We want to have a $d_{1}$ which is large (to facilitate larger $\tau$ ), and at the same time a $\bar{f}_{\mathrm{t}}(\phi)$ which is as close to zero as possible (to stay away from violating (22)). Therefore, the parameter $c_{2}$ should have its maximum value $c_{2}=d_{1} / 2$, as dictated by (27). Similarly, we want $\bar{f}_{\mathrm{n}}(\phi)$ to be as large as possible, and hence because $\cos 4 \phi$ is positive in the considered interval, $b_{2}$ should also be maximal. There are two upper bounds on $b_{2}$, given by (26) and $\bar{f}_{\mathrm{n}}(\phi) \geq 0$, the latter of which turns out to be the most restrictive one. This gives

$$
b_{2}=1+d_{1}-2 \tau .
$$

Having eliminated $b_{2}$ and $c_{2}$, we have thus reduced the optimization problem to 2 parameters, namely $\tau$ and $d_{1}$. To find the parameters that maximize $\tau$, we take the equality sign in (22) and demand that solutions are tangent points. For arbitrary values of the parameters, there is a tangent point in $\phi_{1}=3 \pi / 4$ and two normal intersection points in $\phi_{2}$ and $\phi_{3}$. The intersection points should coincide to turn into a tangent point. Equating $\phi_{2}=\phi_{3}$ gives a relation between the parameters which, after some lengthy but elementary algebra, can be written as

$$
\tau=\frac{1}{2} d_{1}+\frac{1}{3}+\frac{1}{6 \mu} \sqrt{4 \mu^{2}-3 d_{1}^{2}} .
$$

Maximizing this expression for $\tau\left(\mu, d_{1}\right)$ with respect to $d_{1}$ yields that the optimum is 
obtained for

$$
d_{1}=\frac{2 \mu^{2}}{\sqrt{1+3 \mu^{2}}},
$$

so that

$$
\tau_{\max }(\mu)=\frac{1+\sqrt{1+3 \mu^{2}}}{3} .
$$

Inserting these into the original equations gives the value of $\phi$ where the curves touch,

$$
\tan 2 \phi=\frac{\sqrt{1+3 \mu^{2}}-2}{3 \mu},
$$

which was demanded to be in the interval $\left[\frac{7 \pi}{8}, \pi\right]$. This requirement is met for values of $\mu$ satisfying

$$
(0.29 \approx) 1-\frac{1}{2} \sqrt{2} \leq \mu \leq 1 .
$$

This validity is indeed found numerically in figure 5] Note that this range of Coulomb coefficients is the most relevant for real granular materials [30].

For smaller $\mu$ the tangent point is below $\phi=\frac{7 \pi}{8}$, where the $\cos 4 \phi$ is negative, so $b_{2}$ should now be as small as possible: $b_{2}=-(2 \tau-d) / 4$. The resulting quartic equations can be solved using computer algebra, yielding a lenghty expression (not shown), which is plotted as the dashed part of the curve in figure 5] and which coincides with the numerical data.

For $\mu>1$ the above analysis gives a tangent point in the interval $[0, \pi / 12]$, so that the considerations that allowed us to fix $b_{2}$ and $c_{2}$ are no longer valid and we only have the numerical result of figure 5] When the Coulomb coefficient becomes very large, the sustainable shear stress is mostly due to the tangential forces. Because the right hand side of (22) grows linearly with $\mu$, the values of $d_{1}$ and $c_{2}$ can also scale as $\mu$ when $\mu \gg 1$. This leads to an to an asymptotic behaviour of $\tau_{\max }(\mu)$ which is linear in $\mu$. We numerically determined the parameters $a_{1}, b_{2}, c_{2} / \mu, d_{1} / \mu$ for the asymptotic $\bar{f}_{\mathrm{n}}(\phi)$ and $\bar{f}_{\mathrm{t}}(\phi) / \mu$ by optimizing for $d_{1} / \mu$ instead of $\tau$. The resulting asymptote is $\tau=0.376+0.593 \mu$, which plotted as the dotted line in figure 5

\section{Fabric anisotropy}

We now analyze the effect of a fabric anisotropy on the maximum shear stress. The structure of the contact network can be characterized using the fabric tensor $F_{\alpha \beta}=\overline{\mathbf{r}_{\alpha} \mathbf{r}_{\beta} / r^{2}}$, and a fabric anisotropy shows up as a difference between the principal values of this tensor, $F_{1}-F_{2}$. Analogous to the stress tensor, this difference is solely determined by the lowest order coefficient of the Fourier expansion of $\Phi(\phi)$, the contact angle distribution, which we therefore approximate as $\S$

$$
\Phi(\phi)=\frac{1}{\pi}\left(1+p_{1} \sin 2 \phi\right)
$$

One easily shows that $p_{1}=2\left(F_{1}-F_{2}\right)$. The strategy is to again find the maximum possible value for $\tau$, but now for $p_{1} \neq 0$.

Let us note that this truncated form for $\Phi(\phi)$ is a good approximation for systems with a simple shear deformation history [29, 31, although more complex forms are

$\S$ The maximum shear stress is achieved when contact and force anisotropy are "in phase", so that we do not introduce a phase shift in the $\sin 2 \phi$ term of (31). 
Bounds on the shear load of cohesionless granular matter

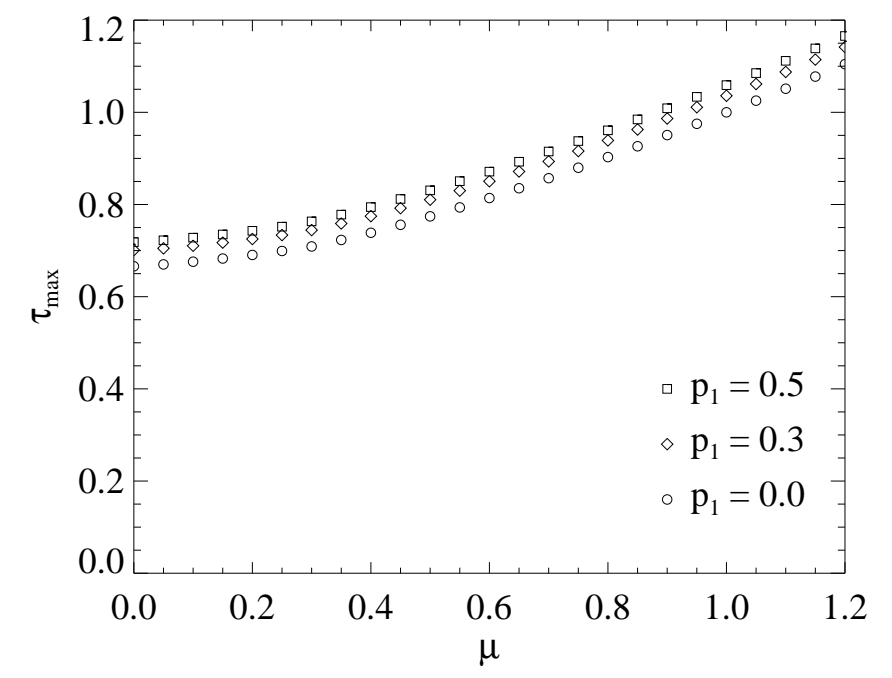

Figure 6. Maximum shear stress $\tau_{\max }$ as a function of friction coefficient $\mu$ for $p_{1}=0,0.3,0.5$.

encountered e.g. for packings formed under gravity [32. As was demonstrated by Troadec et al. [33], the values for $p_{1}$ are bounded by the effect of steric exclusion between neighbouring contacts (in the same way as steric exclusion bounds the force anisotropy, see also Appendix A. Indeed, numerical simulations show that $p_{1}<0.3$, while typically $p_{1}$ is of the order 0.1 [29, 31].

We thus insert the form (31) in the expression for the stress tensor (5), yielding

$$
\begin{aligned}
\sigma_{x x} & =b_{0} / 2+p_{1} a_{1} / 4 \\
\sigma_{y y} & =b_{0} / 2+p_{1} a_{1} / 4 \\
\sigma_{x y} & =\frac{1}{4}\left(a_{1}+d_{1}\right)+\frac{p_{1}}{8}\left(2 b_{0}+c_{2}-b_{2}\right) .
\end{aligned}
$$

This time the second order coefficients do affect the value of the deviatoric stress directly. The idea of the optimization procedure, however, remains the same as in the isotropic case. In this section we present the results of the optimization. The frictionless case, which has an analytic solution, is discussed in more detail in Appendix C

We again eliminate $b_{0}$ by setting $\sigma_{x x}=1 / 2$ in (32). From (34) we then have

$$
\tau=\frac{\sigma_{x y}}{\sigma_{x x}}=\frac{a_{1}\left(2-p_{1}^{2}\right)+2 d_{1}+p_{1}\left(2-b_{2}+c_{2}\right)}{4} .
$$

Working out the optimization for the frictionless case $\left(c_{2}=d_{1}=0\right)$, we get the analytic result

$$
\tau_{\max }=\frac{7 p_{1}+8}{8 p_{1}+12}
$$

as is shown in Appendix C The inclusion of fabric anisotropy hence leads to a small increase of the maximum deviatoric stress; for e.g. $p_{1}=0.3$ we get a $5 \%$ increase. See also the numerical data points on the vertical axis of figure 6

For the complete case, with anisotropic fabric and finite friction coefficient, we have used the same numerical optimization procedure as in section 4.1 The result is 
shown for $p_{1}=0.3,0.5$ in figure [ 6 From this figure one can see that the combined effect of friction and anisotropy can roughly be seen as an addition of their individual effects.

\section{Discussion}

The repulsive-only interaction between dry grains causes the shear stress sustained by granular materials to be bounded. We have derived these upper bounds, $\tau_{\max }$, by finding the extreme shapes of the angle-resolved average force, $\overline{\mathbf{f}}(\phi)$, that are consistent with Coulomb's friction law for individual grains. In the spirit of the Force Network Ensemble, the key question addressed in the present paper is "can such a force network exist?", and we have argued that our analysis represents the strongly hyperstatic limit of the ensemble.

The yielding process in real systems is much more involved and cannot be fully described by the existence criteria used in the paper. However, the analysis does allow to investigate the influence of the micromechanical parameters on the maximum shear stress, in such a way that different aspects can be disentangled. This provides useful complementary information to simulation methods, for which the packing texture cannot be controlled during loading. For example, we have found that an anisotropic fabric is not needed to sustain a large shear stress, and in fact adding fabric anisotropy hardly increases the maximum stress. This suggests that shear-induced textures observed in numerical simulations [29, 31] play a relatively passive role in the stress balance.

Secondly, we analyzed the effect of the microscopic friction coefficient $\mu$, and found that $\tau_{\max }$ does not increase rapidly with $\mu$ in the physically relevant regime (figure 5). One has to be very careful, however, to interpret this result. The presence of friction does have an important influence on the coordination number, which in turn affects the shear resistance: our analysis breaks down close to the isostatic limit, at which the force balance equations become dominant and $\tau_{\max }$ drops to zero [21. Consistent with our analysis, the internal friction measured in simulations of quasistatic shear flows [19, 34] displays only a mild increase is observed when $\mu>0.4$. On the other hand, $\tau$ was found to decrease rapidly as $\mu \rightarrow 0$, which we believe is because the system approaches the isostatic limit $\|$.

Our main conclusion is thus that strongly hyperstatic packings of grains can support an amount of shear stress that is not very sensitive to friction or fabric anisotropy. For systems close to the isostatic limit, however, the dependence of the coordination number on any of these parameters has a more dramatic effect.

Another interesting perspective is that one can use an expansion of $\overline{\mathbf{f}}(\phi)$, as presented in this paper, to estimate the effective elastic moduli of the system, denoted by $C_{\alpha \beta \gamma \delta}$. It has been shown experimentally that these become highly anisotropic when a system is pre-sheared [6, 36. The response to a localized vertical force on the free surface of a granular bed was found not to propagate along the vertical, but along

|| It should be noted that it is not obvious to determine how far from isostaticity (marginal stability) a system is. Two-dimensional systems of frictionless disks in principle become isostatic at $z=3$, but when the microscopic friction coefficient $\mu$ is small, many contacts are fully mobilized (i.e. the frictional force has its maximum value allowed by the Coulomb criterion) 35. These contacts contribute less to the number of degrees of freedom in the force network, since the tangential force component is fixed by the normal force component 1 35. Therefore, these systems are less hyperstatic than one would think on the basis of the coordination number. 
a direction that is tilted towards the major stress axis. This indicates a stiffening of contacts along the major axis that are responsible for the anisotropic elasticity. By fitting the experimentally measured stress profiles Atman et al. 36 obtained the ratio $C_{1111} / C_{2222} \approx 0.67$ of the Young's moduli in the minor (1) and major (2) directions. This finding was remarkably insensitive to frictional properties and roughness of the grains.

The stiffening of contacts can be explained through the nonlinear interaction between particles, which for frictionless Hertzian contacts [37] in three dimensions is $k_{i j} \propto f_{i j}^{1 / 3}$, where $k_{i j}$ is the effective spring-constant of the contact. Assuming that the displacements of the particles are affine - this is reasonable for the strongly hyperstatic packings considered in this paper 38 — one can estimate the elastic moduli as [39, 40] 9

$$
\begin{aligned}
C_{\alpha \beta \gamma \delta} & \propto \int_{0}^{\pi} d \phi \Phi(\phi) \bar{k}(\phi) n_{\alpha} n_{\beta} n_{\gamma} n_{\delta} \\
& \propto \int_{0}^{\pi} d \phi \Phi(\phi)[\bar{f}(\phi)]^{1 / 3} n_{\alpha} n_{\beta} n_{\gamma} n_{\delta} .
\end{aligned}
$$

This allows to systematically explore the effect of stress induced anisotropy in forces or fabric, by again using the Fourier expansions of $\bar{f}(\phi)$ and $\Phi(\phi)$. For the simplest case of isotropic frictionless contacts, one already finds that $C_{1111} / C_{2222}=1-8 \tau / 9+\mathcal{O}\left(\tau^{2}\right)$, so that the experimentally observed ratio of about 0.67 is easily achieved for realistic values of $\tau$.

\section{Acknowledgments}

We thank Martin van Hecke, Thijs Vlugt, Julien Tailleur, Philippe Brunet, Kostya Shundyak, and Wim van Saarloos for numerous discussions. WGE acknowledges financial support from the physics foundation FOM, JHS from a Marie Curie European Fellowship FP6 (MEIF-CT-2006-025104).

\section{Appendix A. Steric exclusion and the coordination number}

We show in section 3 that optimizing $\tau_{\max }$ is equivalent to making the maximum of $\overline{\mathbf{f}}(\phi)$ around the major principal axis as sharp as possible. It has been reported from numerical simulations of realistic systems that in practice this maximum is not very sharp and that typically the highest Fourier contribution to $\overline{\mathbf{f}}(\phi)$ is of order $\cos 4 \phi$ [21, 29] (see also figure 1b). This can be related to the coordination number as follows. Suppose we wish to maximize the forces around the major principal axis, $\phi=\pi / 4$. On a typical grain this can be achieved by putting all stress on the contact closest to this angle. For a randomly oriented grain with $z=6$, the closest contact lies roughly in the range of $\pm \pi / 6$ around the principal direction. Let us imagine that it were possible for a grain to have $z \gg 6$. Now, the contacts closest to the major direction are spread within a much smaller range $\pm \pi / z$, so that much narrower widths of $\overline{\mathbf{f}}(\phi)$ can be achieved. Of course, the situations described above are very extreme because in reality all contacts will be involved in the force equilibrium. In any case,

T The use of $(\bar{f})^{1 / 3}$ instead of $\overline{f^{1 / 3}}$ introduces only a small error since these functions differ mainly around the minor principal axis where the integrand is small anyway. 
these simple examples illustrate that the coordination number introduces a natural scale for the width due to steric exclusion effects.

If the polydispersity is small, only very few grains will have more than six neighbours, and the peaks and valleys in $\overline{\mathbf{f}}(\phi)$ will therefore have a minimum width of approximately $\pi / 3$. This is why a description using only a few terms in a Fourier expansion works, as was already clear from existing numerical results.

\section{Appendix B. Frictionless case at arbitrary order}

In this appendix we analytically solve the linear problem of (20). Due to the symmetry of $\bar{f}_{\mathrm{n}}(\phi)$ we can write

$$
\bar{f}_{\mathrm{n}}(\phi)=1+\sum_{k=1}^{N} q_{k} \sin \left(2 k \phi+\frac{(k-1) \pi}{2}\right) .
$$

Comparing to (16), we find $q_{k}=(-1)^{(k-1) / 2} a_{k}$ for odd $k$, while $q_{k}=(-1)^{k / 2} b_{k}$ for even $k$. In particular, $q_{1}=2 \tau$. The even derivatives can be written as

$$
\frac{\partial^{2 l}}{\partial \phi^{2 l}} \bar{f}_{\mathrm{n}}(\phi)=(-1)^{l} \sum_{k=1}^{N} q_{k}(2 k)^{2 l} \sin \left(2 k \phi+\frac{(k-1) \pi}{2}\right),
$$

and since all sine terms evaluated at $\phi=3 \pi / 4$ yield -1 , we find for $l \neq 0$

$$
\left.\frac{\partial^{2 l}}{\partial \phi^{2 l}} \bar{f}_{\mathrm{n}}(\phi)\right|_{\phi=\frac{3 \pi}{4}}=(-1)^{l+1} \sum_{k=1}^{N} q_{k}(2 k)^{2 l} .
$$

The linear problem of (20) can now be written in the form of a Vandermonde matrix,

$$
\left(\begin{array}{cccc}
1 & 1 & \cdots & 1 \\
x_{1} & x_{2} & \cdots & x_{N} \\
x_{1}^{2} & x_{2}^{2} & \cdots & x_{N}^{2} \\
\cdot & & & \cdot \\
\cdot & & & \cdot \\
\cdot \\
x_{1}^{N-1} & x_{2}^{N-1} & \ldots & x_{N}^{N-1}
\end{array}\right)\left(\begin{array}{c}
q_{1} \\
q_{2} \\
q_{3} \\
\cdot \\
\cdot \\
\cdot \\
q_{N}
\end{array}\right)=\left(\begin{array}{c}
1 \\
0 \\
0 \\
\cdot \\
\cdot \\
\cdot \\
0
\end{array}\right)
$$

with $x_{k}=4 k^{2}$. The inverse $A_{j k}$ of this matrix can be expressed explicitly in terms of Lagrange polynomials as 41]

$$
P_{j}(x)=\prod_{k=1, k \neq j}^{N} \frac{x-x_{k}}{x_{j}-x_{k}}=\sum_{n=1}^{N} A_{j n} x^{n-1} .
$$

We are interested in the solution for $q_{1}=2 \tau$, which for (B.4) simply reads

$$
\begin{aligned}
q_{1} & =A_{11}=P_{1}(0)=\prod_{k=2}^{N} \frac{x_{k}}{x_{k}-x_{1}} \\
& =\prod_{k=2}^{N} \frac{k^{2}}{(k+1)(k-1)}=\frac{2 N}{N+1},
\end{aligned}
$$


so that $\tau=N /(N+1)$, (21). Similarly, the other $q_{k}$ follow from

$$
q_{k}=\prod_{k=1, k \neq j}^{N} \frac{k^{2}}{(k+j)(k-j)} .
$$

\section{Appendix C. Details for the anisotropic frictionless optimization}

We start from the expression for the deviatoric stress in terms of all parameters and

Fourier coefficients, equation (35), where we put $c_{2}=d_{1}=0$ because the tangential force components are zero. This reads

$$
\tau=\frac{a_{1}\left(2-p_{1}^{2}\right)+p_{1}\left(2-b_{2}\right)}{4} .
$$

The physical constraints on $a_{1}$ and $b_{2}$ are the same as for the isotropic case discussed in section 3.1 but the optimization target is now given by (C.1), instead of the $\tau=a_{1} / 2$ we had before. The constraints follow from $\bar{f}_{\mathrm{n}}(\phi)$ and its second derivative being nonnegative at $\phi=3 \pi / 4$ :

$$
\begin{aligned}
& b_{2} \leq 1-a_{1}\left(1+p_{1} / 2\right) \\
& b_{2} \geq-a_{1} / 4 .
\end{aligned}
$$

The solution to this linear program is then found to be

$$
\begin{aligned}
& a_{1}=4 /\left(2 p_{1}+3\right) \\
& b_{0}=3 /\left(2 p_{1}+3\right) \\
& b_{2}=-1 /\left(2 p_{1}+3\right),
\end{aligned}
$$

which corresponds to

$$
\tau_{\max }=\frac{7 p_{1}+8}{8 p_{1}+12}
$$

which is the result stated in equation (36).

\section{References}

[1] Bouchaud J P, 2003 Slow Relaxations and Nonequilibrium Dynamics in Condensed Matter, Proc. of the 2002 Les Houches Summer School, Session LXXVII (EDP Sciences, Les Ulis) pp 131-197

[2] Jaeger H M, Nagel S R and Behringer R P, 1996 Rev. Mod. Phys. 681259 de Gennes P G, 1999 Rev. Mod. Phys. 71374

[3] Radjai F, Jean M, Moreau J-J and Roux S, 1996 Phys. Rev. Lett. 77274

[4] Mueth D M, Jaeger H M and Nagel S R, 1998 Phys. Rev. E 573164

Blair D L, Mueggenburg N W, Marshall A H, Jaeger H M and Nagel S R, 2001 Phys. Rev. E 63041304

[5] Snoeijer J H, Vlugt T J H, van Hecke M and van Saarloos W, 2004 Phys. Rev. Lett. 92054302

[6] Geng J, Reydellet G, Clement E and Behringer R P, 2003 Physica D 182274

[7] Brujic J et al., 2003 Faraday Discussions 123207

[8] Majmudar T S and Behringer R P, 2005 Nature (London) 4351079

[9] Liu A J and Nagel S R, 1998 Nature (London) 39621

[10] Daerr A and Douady S, 1999 Nature (London) 399241

[11] Nedderman R M, 1992 Statics and Kinematics of Granular Materials (Cambridge University Press, Cambridge, England)

[12] Xu N and O'Hern C S, 2006 Phys. Rev. E 73061303

[13] Nowak S, Samadani A and Kudrolli A, 2005 Nature Physics (London) 150

[14] Albert R, Albert I, Hornbaker D, Schiffer P and Barabasi A-L, 1997 Phys. Rev. E 56 R6271 
[15] Kabla A, Debrégeas G, di Meglio J-M and Senden T J, 2005 Europhys. Lett. 71932

[16] Combe G and Roux J-N, 2000 Phys. Rev. Lett. 853628

[17] Oda M, Nemat-Nasser S and Konishi J, 1985 Soils and Foundations 25 No. 3 p 85

[18] Radjai F and Roux S, 2004 The Physics of Granular Media ed Hinrichsen H and Wolf D E (Wiley-VCH, Berlin) p 165

[19] da Cruz F, Emam S, Prochnow M, Roux J-N and Chevoir F, 2005 Phys. Rev. E 72021309

[20] Deboeuf S, Dauchot O, Staron L, Mangeney A and Vilotte J-P, 2005 Phys. Rev. E 72051305

[21] Snoeijer J H, Ellenbroek W G, Vlugt T J H and van Hecke M, 2006 Phys. Rev. Lett. 96098001

[22] Unger T, Kertész J and Wolf D E, 2005 Phys. Rev. Lett. 94178001

[23] Tighe B P, Socolar J E S, Schaeffer D G, Mitchener W G and Huber M L, 2005 Phys. Rev. E 72031306

[24] McNamara S and Herrmann H J, 2004 Phys. Rev. E 70061303

[25] Ostojic S and Panja D, 2006 Preprint cond-mat/0606349

[26] Moukarzel C F, 1998 Phys. Rev. Lett. 811634

[27] Tkachenko A V and Witten T A, 1999 Phys. Rev. E 60687

[28] Goldhirsch I and Goldenberg C, 2002 Eur. Phys. J. E 9245

[29] Radjai F, Wolf D E, Jean M and Moreau J-J, 1998 Phys. Rev. Lett. 8061

[30] See e.g. the Coefficient of Friction reference table on http://www.engineershandbook.com/

[31] Alonso-Marroquín F, Luding S, Herrmann H J and Vardoulakis I, 2005 Phys. Rev. E 71051304

[32] Atman A P F et al., 2005 J. Phys.: Condens. Matter 17 S2391

[33] Troadec H, Radjai F, Roux S and Charmet J C, 2002 Phys. Rev. E 66041305

[34] Unger T, 2006 Preprint cond-mat/0610083

[35] Shundyak K, van Hecke M and van Saarloos W, 2006 Preprint cond-mat/0610205

[36] Atman A P F et al., 2005 Eur. Phys. J. E 1793

[37] Johnson K L, 1985 Contact Mechanics (Cambridge University Press, Cambridge, England)

[38] Ellenbroek W G, Somfai E, van Hecke M and van Saarloos W, 2006 Preprint cond-mat/0604157

[39] Walton K, 1987 J. Mech. Phys. Solids 35213

[40] Bathurst R J and Rothenburg L, 1988 J. Appl. Mech. 5517

[41] Press W H et al., 1992 Numerical Recipes in FORTRAN: The Art of Scientific Computing, 2nd ed. (Cambridge University Press, Cambridge, England) pp 82-89 